\title{
A mechanical model of bacteriophage DNA ejection
}

\author{
Rahul Arun ${ }^{1}$ \\ Adlai E. Stevenson High School, 1 Stevenson Drive, Lincolnshire, IL 60069 \\ Sandip Ghosal ${ }^{2, *}$ \\ Department of Mechanical Engineering and Engineering Sciences and Applied Mathematics, \\ Northwestern University, Evanston, IL 60208 \\ (C)2017 This manuscript version is made available under the CC-BY-NC-ND 4.0 license \\ http://creativecommons.org/licenses/by-nc-nd/4.0/ \\ DOI: 10.1016/j.physleta.2017.05.044
}

\begin{abstract}
Single molecule experiments on bacteriophages show an exponential scaling for the dependence of mobility on the length of DNA within the capsid. It has been suggested that this could be due to the "capstan mechanism" - the exponential amplification of friction forces that result when a rope is wound around a cylinder as in a ship's capstan. Here we describe a desktop experiment that illustrates the effect. Though our model phage is a million times larger, it exhibits the same scaling observed in single molecule experiments.

Keywords: bacteriophage, cell mechanics, capstan model, Coulomb-Amonton law, DNA translocation, nanoscale friction
\end{abstract}

\section{Introduction}

A bacteriophage is a virus that infects bacterial cells. Like all viruses, they lack the machinery to express the genetic information that they contain. Once

\footnotetext{
* Corresponding author

Email address: s-ghosal@u.northwestern.edu (Sandip Ghosal)

${ }^{1}$ presently at: California Institute of Technology, Pasadena, CA

2phone: (847)467-5990 fax: (847)491-3915 (Sandip Ghosal)
} 
inside their hosts, they "hijack" the cell's gene transcription mechanism to repli-

cate themselves. Phages are essentially made of two components, a protein capsid and the DNA (or RNA in case of RNA phages) that it encloses [1]. Infection is initiated by the phage attaching to the cell membrane followed by injection of the DNA into the cell. The capsid remains outside attached to the cell membrane.

There are several known mechanisms that phages use to inject genetic material into hosts $[2,3,4,5]$. In most double stranded DNA (dsDNA) phages, a fast ejection on the timescale of seconds can be achieved by the release of elastic and electrostatic energy of the coiled up DNA confined within the viral capsid [6]. This is plausible because dsDNA has a persistence length $\sim 50 \mathrm{~nm}$ 15 and a very long strand (e.g. 48.5 kilobase pair or $\sim 17 \mu \mathrm{m}$ for the $\lambda$ phage) is packed into a capsid of diameter of the order of the DNA persistence length. In fact, for the $\lambda$-phage, the internal pressure in the phage capsid is estimated to be $\sim 50-100$ atmospheres [6]. It is nevertheless unlikely that this mechanism alone can explain DNA injection in all dsDNA phages. First, the driving pressure decreases rapidly as DNA empties the capsid; second, the interior of bacterial cells have a relatively high osmotic pressure $\sim 25$ atmospheres that would significantly retard DNA entry. In some phages, the DNA ejection takes place in two steps. Initially, a part of the DNA is injected by this "coiled spring" mechanism. Subsequently, the inserted DNA is expressed to sythesize molecular motors which then reel in the remainder of the DNA by an ATP driven pulling action [7].

In order to understand the ejection process without too many layers of complexity, in vitro experiments have been designed [8] where $\lambda$ phages are induced to eject their DNA into the surrounding buffer in the absence of any host cells. 30 Here, it is indeed the elastic and electrostatic energy of the tightly coiled DNA within the capsid that drives the ejection. Furthermore, in the case of the $\lambda$ phage, the ejection takes place as a single continuous process without pauses and stops. The driving force can be calculated from first principles by regarding the DNA as a charged semi-flexible rod $[9,10]$. The results of such calculations have 
been confirmed by experiments where the DNA is "stalled" after partial ejection by raising the osmotic pressure in the bath $[11,12]$. The speed of ejection, however, is determined not just by the stored potential energy in the capsid but also by the mechanisms of dissipation in the system, and this is more difficult to calculate. Dissipation also plays a role in the opposite process of ATP driven 40 packaging of DNA into the capsid. In that situation, it determines the time scale for reaching thermodynamic equilibrium. If the packaging rate is fast relative to this time scale then the DNA can take up a more disordered configuration than the ordered one that corresponds to the free energy minimum. On subsequent release from confinement, it encounters a higher frictional resistance as can be 45 demonstrated in experiments as well as numerical simulations [13, 14].

It has been proposed [15] that the dissipation arises from frictional forces between the DNA and the capsid wall and between neigboring strands as the helically wound DNA slides out of confinement. The frictional interaction is modeled by the classical Coulomb-Amonton laws $[16,17,18]$ while disregard${ }_{50}$ ing the precise microscopic mechanisms. This is a plausible assumption as the Coulomb-Amonton laws have been found to hold for nanoscale systems, though the underlying mechanism is quite different from that of the classical picture involving interlocking asperities $[19,20,21]$. The Coulomb-Amonton laws when combined with the equilibrium equations of an elastic rod lead to the conclu55 sion that the tension in the rod increases exponentially with the length of DNA confined in the capsid [15]. This exponential amplification of tension is a well known fact in mechanics and is known as the "capstan principle". It is the principle of operation of a winch and various other familiar engineering innovations. The name derives from the cylinder or "capstan" used since antiquity for mooring ships. Another example of the exponential amplification of friction forces is the extraordinary holding power of phone books with interleaved pages [22, 23]. When applied to the ejection of dsDNA from phages, the capstan principle leads to the conclusion that the ejection velocity per unit driving force (the mobility) should decrease exponentially with the amount of DNA confined within the ${ }_{65}$ capsid at any given instant. This is consistent with in vitro experiments on 

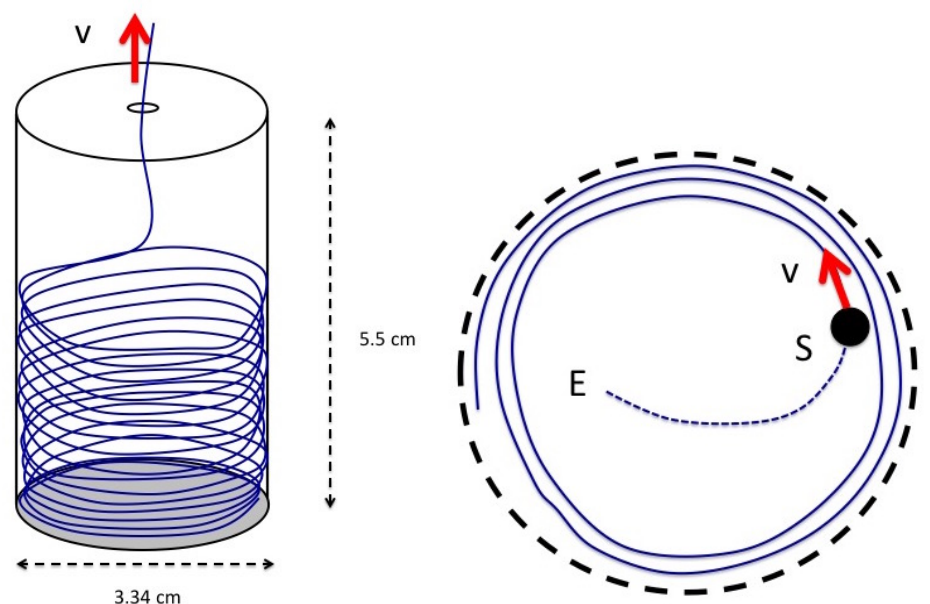

Figure 1: Left: Schematic drawing of the mechanical model of a bacteriophage (see Supplementary Materials for photograph and video of experiment). Right: Top view showing the spiral arrangement of the nylon fiber within the container. The fluid resistance on the trailing end $\mathrm{SE}$ is represented by the drag $(k v)$ on a single hypothetical particle indicated here by the filled circle.

$\lambda$-phages [15].

Since the capstan mechanism also operates on macroscopic scales, it should be possible to demonstrate the exponential decrease of mobility on confined length using a centimeter scale model of the phage. In this paper we describe a table top experiment performed using everyday objects that show this dependance. The paper is organized as follows. The main theoretical ideas relating to the capstan effect in phages are summarized in section 2. In section 3 a desktop experiment illustrating the mechanics of DNA ejection from phages is described. In section 4 the data from the experiment is analyzed in the light of the theoretical ideas discussed earlier in section 2. The significance of the experiment in the context of the DNA-phage problem and its limitations are discussed in section 5. Finally, the main conclusions are summarized in section 6 . 


\section{The Capstan Model}

Figure 1 is a schematic diagram of our experimental set up using a plastic

80 DNA). The question that we wish to answer is the following: how does the length of fiber remaining within the capsid $(x)$ vary with time $(t)$ during the ejection process?

The driving force $F$ in the ejection process can be obtained from an energy argument. The driving force due to elasticity of the fiber is the same as the inward directed external force $F$ that must be applied to the DNA at the capsid exit to prevent it from exiting. The amount of this force can be calculated from the principle of virtual work

$$
F d x=d \mathcal{F}
$$

where $\mathcal{F}(x)$ is the free energy of the DNA. From the theory of linear elasticity, the elastic bending energy of the DNA may be written as

$$
\mathcal{F}(x)=\int_{0}^{x} \frac{E I}{2} \kappa^{2}(s) d s
$$

where $E$ is the Young's modulus of the material, $I$ is the area moment of inertia of the fiber about the neutral plane, and, $\kappa(s)$ is the local radius of curvature of the centerline at a distance ' $s$ ' from the capsid exit. In our mechanical model, the volume of nylon fiber inserted is only a small fraction of the capsid volume so that $\kappa(s) \approx 1 / R, R$ being the radius of the cylindrical container. Thus, under these low packing conditions,

$$
\mathcal{F}(x) \approx \frac{E I}{2 R^{2}} x,
$$

so that the driving force is

$$
F=\mathcal{F}^{\prime}(x)=\frac{E I}{2 R^{2}} .
$$

In the absence of an external stalling force, a tension

$$
T(0)=F=\frac{E I}{2 R^{2}}
$$


acts on the DNA at the capsid entrance that must be balanced by frictional resistance on the filament arising from within the capsid.

If we assume that the fiber may be described by the equations of elastic equilibrium of a beam and frictional forces are governed by the Coulomb-Amonton law with coefficient of kinetic friction $\left(\mu_{k}\right)$, then ${ }^{3}[15]$

$$
T(s)=T(0) \exp \left(\frac{\mu_{k} s}{R}\right)=\frac{E I}{2 R^{2}} \exp \left(\frac{\mu_{k} s}{R}\right),
$$

neglecting any reduction in coil radius due to high packing fractions. Equation (6) is the Euler-Eytelwein formula. It is well known for the problem of a flexible or semi-flexible string wrapped around a cylinder [24], but is also applicable, as in this case, to the "inverted" problem of a semi-flexible rod confined within a hollow cylinder [15].

The constant curvature configuration of the beam cannot extend to the extremity E. This is because the internal bending moment is proportional to the curvature and the internal bending moment must vanish at the free boundary E. What this means is that the fiber will lose contact with neighboring strands at some intermediate point S (Figure 1) before E. Determining the true configuration of the fiber under these circumstances then becomes a difficult free boundary problem since neither the location of E nor the shape of the section $\mathrm{SE}$ is known apriori. An analogous problem where a beam is pushed onto a hard surface from a point a fixed distance above it has been analyzed recently [25] and is shown to exhibit hysteresis of shape controlled by the static friction coefficient. In Brownian molecular dynamic simulations of bead-chain models, helical arrangements are spontaneously generated except for the trailing ends of the chain [26]. In order to avoid the complexity of having to solve free boundary problems, we introduce a "lumped parameter" model for the resistance (possibly arising out of a combination of frictional and hydrodynamic forces) on the trailing end of the DNA that is not part of the helical arrangement. We do this by supposing that the helically wound fiber is terminated (point S in Figure 1)

\footnotetext{
${ }^{3}$ Eq. (13) of [15] contains an error: the factor of $2 \pi$ in the exponent is superfluous.
} 
by a bead that experiences a hydrodynamic drag $k v$ where $k>0$ is a drag coefficient. Thus,

$$
T(x)=\frac{E I}{2 R^{2}} \exp \left(\frac{\mu_{k} x}{R}\right)=k v=-k \frac{d x}{d t} .
$$

The solution of this differential equation then gives the time dependence of the length $x$ remaining within the capsid

$$
x=L-\frac{R}{\mu_{k}} \ln \left[1+\frac{E I \mu_{k}}{2 k R^{3}} \exp \left(\frac{\mu_{k} L}{R}\right) t\right]
$$

where $L$ is the length of fiber initially in the capsid ignoring the short section SE. At short times, $t \rightarrow 0$, we have

$$
x=L-v_{m} t
$$

where

$$
v_{m}=\frac{E I}{2 k R^{2}} \exp \left(\frac{\mu_{k} L}{R}\right) .
$$

Eq. (8) may be rewritten as

$$
x=L-\frac{R}{\mu_{k}} \ln \left(1+\frac{\mu_{k}}{R} v_{m} t\right)
$$

and on differentiation,

$$
v=-\frac{d x}{d t}=\frac{v_{m}}{1+t / \tau}
$$

where

$$
\tau^{-1}=\mu_{k} v_{m} / R .
$$

Thus, the velocity decreases monotonically from a maximum of $v_{m}$ on a time scale $\tau$. The total time of ejection $T$ is obtained by setting $t=T$ and $x=0$ in equation (11)

$$
\frac{L}{v_{m} \tau}=\ln \left(1+\frac{T}{\tau}\right) .
$$

Here we have assumed that the length of the residual portion of the fiber that is not ejected may be taken as equal to the length SE in Figure 1. 


\section{Methods}

The bacteriophage model consists of a cylindrical (height, $\mathrm{h}=5.5 \mathrm{~cm}$, radius, $\mathrm{R}=1.67 \mathrm{~cm}$ ) polyethylene film canister (Fuji $35 \mathrm{~mm}$, type: frosted clear, material: HDPE body, LDPE cap) representing the capsid and a nylon fishing line (Berkley Trilene Big Cat Monofilament Line, $0.55 \mathrm{~mm}$ average diameter, $13.6 \mathrm{~kg}$ break strength) representing the viral DNA. A centered $2 \mathrm{~mm}$ diameter hole was drilled into the top of the film canister through which the monofilament was packaged and ejected. Pieces of filament were pre-cut to the following lengths for the experiment $L_{\max }=50,100,150,200$ and $290 \mathrm{~cm}$. The capsid was loaded by inserting one end of a filament into the container and slowly pushing in a pre-cut section of filament. As soon as the length of filament exceeded some multiple of the canister height, the filament spontaneously arranged itself in a helical pattern against the inside wall of the canister just as observed in Brownian molecular dynamic simulations of DNA-phage systems [26].

155 The filament was confined by covering the exit hole with the thumb and released by removing the thumb. The event was recorded with a movie camera (Drift Innovation HD170 Action Camera, maximum frame rate of $60 \mathrm{fps}$ and resolution 720p) and the ejection time was measured with a stop watch (iPhone 5, Apple Inc.) measuring to $0.01 \mathrm{~s}$. The time between the instant the hole was opened and the instant the filament stopped moving was recorded. In all cases, the ejection stopped with a variable amount of residual length remaining in the capsid. The recorded value of $L$ was taken as the actual length of filament pushed out of the capsid, that is, the true length $\left(L_{\max }\right)$ minus the residual length. A similar behavior is also seen in the $\lambda$ phage in vitro experiment [8] where there is a long pause between the time when the ejection stops and the time at which the DNA finally separates from the capsid. The experiment was repeated several times with each filament section and the ejected length $L$ and ejection time $T$ was recorded. This data is shown in Section 3, Table 1 of the accompanying Supplementary Material. It is seen that there is a spread in the measured values of $L$ and $T$ by an amount $\Delta L \sim \pm 2 \mathrm{~cm}$ and $\Delta T \sim \pm 0.5 \mathrm{~s}$. 
The experiment was then repeated by submersing the system first in filtered water (Ice Mountain $100 \%$ Natural Spring bottled drinking water) and then in glycerin (Soap Expressions 100 \% Vegetable Based). In each case, care was taken to ensure that the liquid completely filled the container without visible air bubbles. The fiber was pre-loaded prior to flooding the container with the fluid. The corresponding ejected length $(L)$ and ejection time $(T)$ data are recorded in Section 3, Table 2 (water) and Table 3 (glycerin) of the accompanying Supplementary Material. The displayed data are an average over four separate runs (three, in the case of the longest fiber length) of the experiment. A still image of the loaded capsid (Section 1) and a slow motion video of the capsid firing (Section 2) are also included.

\section{Results}

The ejection process appeared qualitatively similar in all three media except for the speed of ejection. In water, the process was slowed down by about a factor of five relative to that in air and by about a factor of almost two hundred in glycerin. Notwithstanding the widely different translocation times, equation (14) provided a good fit to the data in all cases as shown in Figure 2 using the similarity variables $L /\left(v_{m} \tau\right)$ and $T / \tau$. For each data set, the two parameters $v_{m} \tau$ and $\tau$ were chosen to obtain the best fit to equation (14). The fits were arrived at iteratively by trial and error and goodness of fit was decided visually. The best fit parameter values thus obtained are summarized in Table 1. In each of the three cases, the value of the kinetic friction coefficient $\mu_{k}$ inferred from

\begin{tabular}{|c|c|c|c|c|}
\hline & Unit & Air & Water & Glycerin \\
\hline$v_{m}$ & $\mathrm{~cm} / \mathrm{s}$ & 140 & 70 & 1.6 \\
$\tau$ & $\mathrm{s}$ & 2.0 & 1.9 & 74 \\
$\mu_{k}$ & - & 0.006 & 0.013 & 0.014 \\
\hline
\end{tabular}

Table 1: Scaling parameters $v_{m}$ and $\tau$ for air, water and glycerin ejections for the data shown in Figure 2. The corresponding friction coefficient $\mu_{k}$ is evaluated from equation (13). 


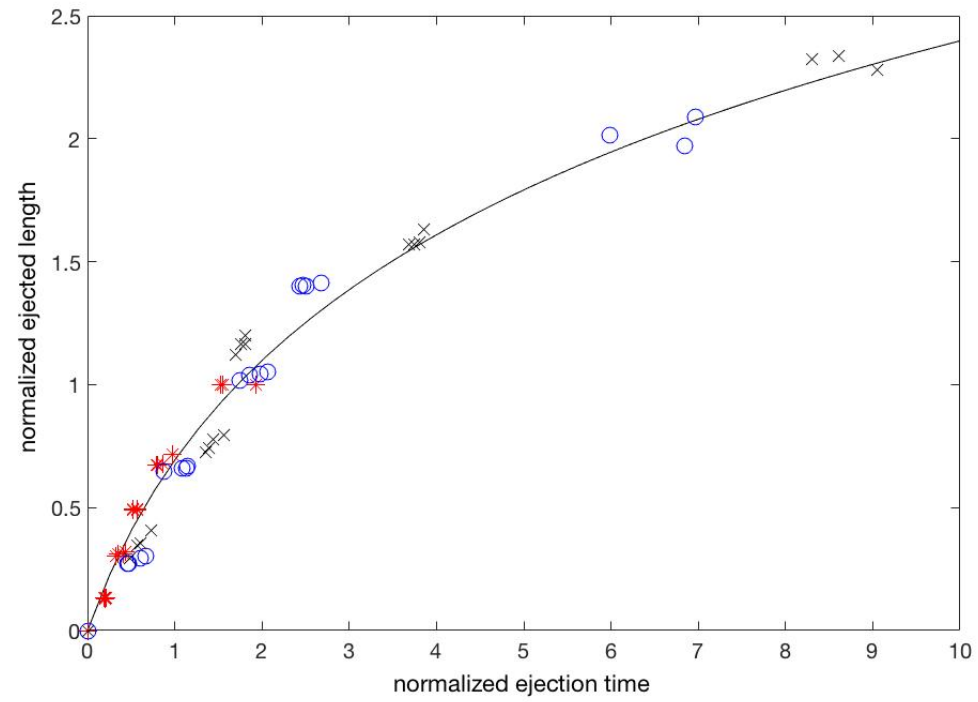

Figure 2: The normalized ejected length $L /\left(v_{m} \tau\right)$ plotted against the normalized ejection time $T / \tau$ for air (star), water (circle) and glycerin (cross) ejections. The solid line is equation (14).

equation (13) is also noted. In all three cases, notwithstanding the wide range of velocity and time scales, the friction coefficients are of similar magnitude. Also, the inferred value is not widely different from published values for lubricated polymer materials, though a detailed comparison will not be justified due to the rough nature of these experiments.

\section{Discussion}

The desktop experiment described here is only meant to illustrate the part of the DNA-phage system behavior relating to the capstan mechanism and is not necessarily an appropriate model for all aspects of the DNA ejection problem in phages. Indeed the two systems differ widely in spatial scales as well as in the details of the physical interactions that control their behavior.

An important difference between the current experiment and the real DNAphage system is the extent of packing of the capsids. In $\lambda$-phage, the volume of the polymer relative to the maximum available capsid volume (the packing 
fraction, $f$ ) is in the range $0.4-0.5[27]$. In the mechanical model presented here, the packing fraction is easily estimated from the geometric parameters in section $3, f \approx 0.002-0.015$, much smaller than the actual values encountered in phages. The behavior at high packing fractions has been explored recently using macroscopic mechanical models to represent the phage-DNA system [28, 29, 30]. The focus of these investigations is the shape acquired by the packed DNA inside the capsid rather than the dynamics of ejection studied here. Also, the metal wires used have significantly higher plasticity than the nylon fibers used in the present experiments.

The low packing fractions used here is also responsible for an important qualitative difference between our model and the real phage system. Measured ejection velocities in the $\lambda$ phage when plotted as a function of the DNA remaining within the capsid show a characteristic unimodal shape. That is, the escape velocity first increase as the capsid starts to empty, reaches a maximum at an intermediate stage and finally decreases. This is because the velocity $v=F(x) m(x)$ where the mobility $m(x) \propto \exp (-b x)(b>0)$ but the driving force $F$ is an increasing function of $x$ since the capsid pressure increases as the capsid is tightly packed. In fact, when the steric constraint is accounted for, ${ }_{225} F(x) \propto\left(1-x / x_{m}\right)^{-1}$ where $x_{m}$ is the maximum length of DNA that the capsid can accommodate $[9,10]$. In the experiment described here, the volume fraction of fiber inserted was small compared to the maximum capacity of the capsid, to avoid jamming and other practical complications. Thus, $F(x)$ is a constant given by equation (4) so that the velocity decreases monotonically rather than show a peak at an intermediate time.

The scale of the DNA-phage problem is such that any hydrodynamic interactions would be firmly in the Stokesian regime, that is, the Reynold's number $R e=\rho v d / \mu$, where $\rho$ is the fluid density, $\mu$ is the viscosity, $v$ is a characteristic velocity and $d$ is a characteristic length, is essentially zero. In our mechanical 235 model, using the exit hole diameter $(2 \mathrm{~mm})$ as a length scale and the maximumum value of the ejection velocity $v_{m}$ as the velocity scale, we find $R e=374$, 1 and 0.02 respectively for ejections in air, water and glycerin. Thus, only 
the glycerin experiments truly represent the hydrodynamic conditions in the DNA-phage system. However, we find that the scaling law, equation (14), holds irrespective of the working fluid though the ejection velocity itself depends on fluid viscosity. This is consistent with the capstan model that attributes the exponential dependence of mobility on filament length to interstrand friction, and fluid viscosity only plays an incidental role [15].

The pressure within the viral capsid ejecting the DNA arises from a combination of the bending energy of the DNA and the electrostatic self-repulsion between strands. These two factors contribute about equally to the potential energy of the confined DNA [6]. The electrostatic part of the potential energy can be readily tuned by changing the ionic composition of the solvent and thereby changing the Debye length. At high ionic concentrations and in the presence of polyvalent ions such as $\mathrm{Mg}^{2+}$ the DNA backbone charge is almost perfectly shielded, reducing the electrostatic self-repulsion to almost zero. Ionic composition changes are found to affect the ejection speed in experiments [8] mainly through its effect on the capsid pressure. Numerical simulations suggest [31] that the topology of the DNA configuration during the packaging phase can depend on the ionic composition of the bath. When the packaged DNA is allowed to eject freely, the ejection speed could in turn depend on the topology of the packaged strand [32]. These aspects of the problem of course cannot be accessed using our mechanical model as the stored potential energy in our nylon fiber is purely elastic; unlike DNA, it does not have an electrostatic component.

\section{Conclusion}

Recent advances in single molecule observation and manipulation have made it possible to study the packaging [33] and ejection [8] of DNA from phages with in vitro techniques. Such experiments have resulted in quantitative data that require explanations and interpretations. These problems belong to the "mesoscale" where the familiar classical mechanics of solids and fluids may still be applied but often in association with effects such as statistical fluctuations 
and Debye layer physics that are peculiar to small scale systems. In this paper, we consider one such problem, bridging a gap of almost seven orders of magnitude to take ideas very familiar in marine engineering (winches and capstans) and applying them in the realm of cell biology (phages and DNA).

The specific single molecule experiment of interest here involves the ejection of DNA by the $\lambda$ phage into the surrounding buffer. It was shown that the experimental data on ejection velocity is consistent with a frictional resistance law that has an exponential dependence on the length of DNA in the capsid. A possible mechanism for this was suggested [15] based on the "capstan effect", where the helical geometry of the DNA together with the Coulomb-Amonton friction law results in an exponential amplification of friction with increasing turns.

The conditions required for the capstan mechanism can be simulated in a large scale system just as easily as in the bacteriophage. In this paper we have presented results from a desktop experiment that mimicks the process of DNA ejection from bacteriophages. The measured ejection time as a function of ejected length was found to be consistent with a scaling law derived on the basis of the capstan model. The scaling law was found to be independent of the Reynold's numbers in the range $\sim 0.01-300$.

This research did not receive any specific grant from funding agencies in the public, commercial, or not-for-profit sectors.

\section{Acknowledgement}

We wish to thank Prof. Sigurdur Thoroddsen (King Abdullah University of Science and Technology) for helpful suggestions and the Department of Mechanical Engineering, Northwestern University for loan of the video camera. 


\section{References}

\section{References}

[1] C. M. Knobler and W. M. Gelbart. Physical Chemistry of DNA Viruses, Annual Review of Physical Chemistry, 60(1):367-83, May 2009.

[2] I. J. Molineux. Fifty-three years since Hershey and Chase; much ado about pressure but which pressure is it? Virology, 344(1):221-229, January 2006.

[3] M. M. Inamdar, W. M. Gelbart, and R. Phillips. Dynamics of DNA Ejection from Bacteriophage. Biophysical Journal, 91(2):411-420, July 2006.

[4] P. Grayson and I. J. Molineux. Is phage DNA 'injected' into cells - biologists and physicists can agree. Current Opinion in Microbiology, 10(4):401-409, August 2007.

[5] I. J. Molineux and D. Panja. Popping the cork: mechanisms of phage genome ejection. Nature Reviews Microbiology, 11(3):194-204, March 2013.

[6] M. De Frutos, A. Leforestier, and F. Livolant. Relationship between the genome packing in the bacteriophage capsid and the kinetics of dna ejection. Biophysical Reviews and Letters, 09(01):81-104, November 2013.

[7] V. González-Huici, M. Salas, and J. M. Hermoso. The push-pull mechanism of bacteriophage $\phi 29$ DNA injection. Molecular Microbiology, 52(2):529540, March 2004.

[8] P. Grayson, L. Han, T. Winther, and R. Phillips. Real-time observations of single bacteriophage $\lambda$ DNA ejections in vitro. Proceedings of the National Academy of Sciences of the United States of America, 104(37):14652-14657, September 2007.

315

[9] P. K. Purohit, J. Kondev, and R. Phillips. Mechanics of DNA Packaging in Viruses. Proceedings of the National Academy of Sciences, 100(6):31733178, March 2003. 
[10] P. K. Purohit, M. M. Inamdar, P. D. Grayson, T. M. Squires, J. Kondev, and R. Phillips. Forces during Bacteriophage DNA Packaging and Ejection. Biophysical Journal, 88(2):851-866, February 2005.

[11] A. Evilevitch, L. Lavelle, C. M. Knobler, E. Raspaud, and W. M. Gelbart. Osmotic pressure inhibition of DNA ejection from phage. Proceedings of the National Academy of Sciences, 100(16):9292-9295, August 2003.

[12] A. Evilevitch, M. Castelnovo, C. M. Knobler, and W. M. Gelbart. Measuring the Force Ejecting DNA from Phage. J. Phys. Chem. B, 108(21):6838$6843,2004$.

[13] Z. T. Berndsen, N. Keller, S. Grimes, P. J. Jardine, and D. E. Smith. Nonequilibrium dynamics and ultraslow relaxation of confined DNA during viral packaging. Proceedings of the National Academy of Sciences, 111(23):8345-8350, June 2014.

[14] L. R. Comolli, A. J. Spakowitz, C. E. Siegerist, P. J. Jardine, S. Grimes, D. L. Anderson, C. Bustamante, and K. H. Downing. Three-dimensional architecture of the bacteriophage $\phi 29$ packaged genome and elucidation of its packaging process. Virology, 371(2):267-277, February 2008.

[15] S. Ghosal. Capstan Friction Model for DNA Ejection from Bacteriophages. Physical Review Letters, 109(24):248105, December 2012.

[16] C. Marone. Laboratory-Derived Friction Laws and Their Application to Seismic Faulting. Annual Review of Earth and Planetary Sciences, 26(1):643-696, 1998.

[17] B. N. J. Persson. Sliding Friction: Physical Principles and Applications. Springer, July 2000.

[18] F. P. Bowden and D. Tabor. The Friction and Lubrication of Solids. Oxford University Press, April 2001. 
[19] Y. Mo, K. T. Turner, and I. Szlufarska. Friction laws at the nanoscale. Nature, 457(7233):1116-1119, February 2009.

[20] J. Gao, W. D. Luedtke, D. Gourdon, M. Ruths, J. N. Israelachvili, and U. Landman. Frictional Forces and Amontons' Law: From the Molecular to the Macroscopic Scale. The Journal of Physical Chemistry B, 108(11):34103425, March 2004.

[21] E. Gnecco, R. Bennewitz, T. Gyalog, C. Loppacher, M. Bammerlin, E. Meyer, and H.-J. Güntherodt. Velocity Dependence of Atomic Friction. Physical Review Letters, 84(6):1172-1175, February 2000.

[22] H. Alarcón, T. Salez, C. Poulard, J.-F. Bloch, É. Raphaël, K. DalnokiVeress, and F. Restagno. Self-Amplification of Solid Friction in Interleaved Assemblies. Physical Review Letters, 116(1):015502, January 2016.

[23] K. Dalnoki-Veress, T. Salez, and F. Restagno. Why can't you separate interleaved books? Physics Today, 69(6):74-75, May 2016.

[24] I. M. Stuart. Capstan equation for strings with rigidity. British Journal of Applied Physics, 12(10):559-562, October 1961.

[25] T. G. Sano, T. Yamaguchi, and H. Wada. Slip Morphology of Elastic Strips on Frictional Rigid Substrates. Physical Review Letters , (118):178001, April 2017.

[26] J. Kindt, S. Tzlil, A. Ben-Shaul, and W. M. Gelbart. DNA Packaging and Ejection Forces in Bacteriophage. Proceedings of the National Academy of Sciences, 98(24):13671-13674, November 2001.

[27] P. Grayson, A. Evilevitch, M. M. Inamdar, P. K. Purohit, W. M. Gelbart, C. M. Knobler, and R. Phillips. The effect of genome length on ejection forces in bacteriophage lambda. Virology, 348(2):430-436, May 2006.

[28] M. A. F. Gomes, V. P. Brito, and M. S. Araújo. Geometric properties of crumpled wires and the condensed non-solid packing state of very long 
molecular chains. Journal of the Brazilian Chemical Society, 19(2):293-298, 2008.

[29] T. A. Sobral, M. A. F. Gomes, N. R. Machado, and V. P. Brito. Unpacking of a Crumpled Wire from Two-Dimensional Cavities. PLOS ONE, 10(6):e0128568, June 2015.

[30] V. H. de Holanda and M. A. F. Gomes. Scaling, crumpled wires, and genome packing in virions. Physical Review E, 94(6):062406, December 2016.

[31] I. Ali and D. Marenduzzo. Influence of ions on genome packaging and ejection: a molecular dynamics study. The Journal of Chemical Physics, 135(9):095101-095101, September 2011.

[32] D. Marenduzzo, C. Micheletti, E. Orlandini, and D. W. Sumners. Topological friction strongly affects viral DNA ejection. Proceedings of the National Academy of Sciences, 110(50):20081-20086, December 2013.

[33] D. E. Smith, S. J. Tans, S. B. Smith, S. Grimes, D. L. Anderson, and C. Bustamante. The bacteriophage $\phi 29$ portal motor can package DNA against a large internal force. Nature, 413(6857):748-752, October 2001. 


\section{List of supplementary material}

The following supplementary material accompanies this paper

390

1. Photograph illustrating experimental set up.

2. Video of experiment in progress.

3. Tabular data of measured ejection times. 


\section{Supplementary Material* \\ "A mechanical model of bacteriophage DNA ejection" \\ Rahul Arun \& Sandip Ghosal}

\section{Photograph illustrating experimental set up}

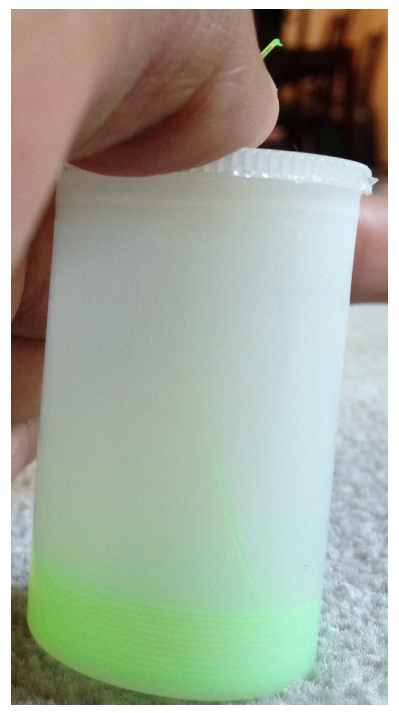

Figure 1: Photograph of mechanical model. Capsid - $35 \mathrm{~mm}$ Fuji film canister (Type: frosted clear, Body: HDPE, Cap: LDPE). DNA - Nylon Monofilament fishing line, brand: Berkley Trilene Big Cat 0.022 in $(0.55 \mathrm{~mm})$ average diameter, break strength $30 \mathrm{lb}$ (13.6 $\mathrm{kg})$.

*(C)2017 This manuscript version is made available under the CC-BY-NC-ND 4.0 license http://creativecommons.org/licenses/by-nc-nd/4.0/ DOI: 10.1016/j.physleta.2017.05.044 


\section{Video of experiment in progress}

Video file: VideoAirSlowMo1.mov

(available from corresponding author upon request)

\section{Video Descriptor:}

Video of nylon monofilament with $5 \mathrm{~cm}$ color bands (for enhanced visibility) ejected from the film canister representing the viral capsid. The medium in this case is air. The "Drift Innovation HD170" Action Camera was used with a Frame Rate of 60 FPS and a resolution of $720 \mathrm{p}$. The video is slowed down by a factor of 8 . 


\section{Tabular data of measured ejection times}

The data points shown in Figure 2 of the paper are as follows:

\begin{tabular}{|c|c|}
\hline Length $(\mathrm{cm})$ & Time $(\mathrm{s})$ \\
\hline 0.0 & 0.00 \\
36.0 & 0.40 \\
37.0 & 0.38 \\
37.0 & 0.41 \\
37.0 & 0.43 \\
85.0 & 0.66 \\
87.0 & 0.71 \\
87.0 & 0.81 \\
90.0 & 0.86 \\
137.0 & 1.05 \\
137.0 & 1.13 \\
139.0 & 1.03 \\
139.0 & 1.13 \\
188.0 & 1.60 \\
189.0 & 1.58 \\
190.0 & 1.73 \\
200.0 & 1.96 \\
279.6 & 3.05 \\
279.6 & 3.85 \\
280.3 & 3.10 \\
\hline
\end{tabular}

Table 1: Ejected length vs time for ejections in air 


\begin{tabular}{|c|c|}
\hline Length $(\mathrm{cm})$ & Time $(\mathrm{s})$ \\
\hline 0.0 & 0.00 \\
36.0 & 0.88 \\
36.0 & 0.90 \\
39.0 & 1.15 \\
40.0 & 1.26 \\
86.0 & 1.66 \\
88.0 & 2.05 \\
88.0 & 2.15 \\
89.0 & 2.18 \\
135.0 & 3.32 \\
138.0 & 3.53 \\
139.0 & 3.75 \\
140.0 & 3.93 \\
186.0 & 4.63 \\
186.0 & 4.76 \\
187.0 & 4.70 \\
188.0 & 5.08 \\
261.9 & 13.01 \\
267.8 & 11.38 \\
278.0 & 13.23 \\
\hline
\end{tabular}

Table 2: Ejected length vs time for ejections in water 


\begin{tabular}{|c|c|}
\hline Length $(\mathrm{cm})$ & Time $(\mathrm{s})$ \\
\hline 0.0 & 0.00 \\
35.0 & 35.91 \\
41.0 & 42.40 \\
42.0 & 44.60 \\
48.0 & 54.40 \\
86.0 & 100.10 \\
88.0 & 103.35 \\
92.0 & 106.63 \\
94.0 & 115.51 \\
133.0 & 125.16 \\
138.0 & 130.16 \\
138.0 & 133.65 \\
142.0 & 133.99 \\
186.0 & 272.18 \\
186.0 & 276.76 \\
187.0 & 281.21 \\
193.0 & 284.87 \\
270.1 & 670.00 \\
275.1 & 614.00 \\
276.8 & 637.00 \\
\hline
\end{tabular}

Table 3: Ejected length vs time for ejections in glycerin 